\title{
IDENTIFICATION OF MONOCLONAL IMMUNOGLOBULINS BY IMMUNOFIXATION ELECTROPHORESIS - SOME QUESTIONS
}

\author{
Miloš Tichý \\ Purkyně Military Medical Academy, Hradec Králové: Second Department of Internal Medicine
}

Summary: In the series of 2413 paraproteins analyzed by immunoelectrophoresis during 30 years, doubled paraproteinemias were found 42 times, i.e. a frequence of $1.7 \%$. In last two years we have found 202 paraproteins by means of immunofixation electrophoresis and within this group multiple paraproteinemias was found 21 times, i.e. a frequence of $10.4 \%$.

Key words: Monoclonal immunoglobulin; Immunofixation; Multiple paraproteinemias

Over nearly the last 30 years the standard investigation method for the identifying of monoclonal immunoglobulins (paraproteins) was based upon electrophoresis of serum proteins and in case positive M-gradient a subsequent immunoelectrophoresis. Now, electrophoretic systems of a high quality, for example using agarose as a dividing medium, are able to identify small M-gradients in concentrations of about $1 \mathrm{~g} / 1$. Immunoelectrophoresis in clinical laboratories has lately been practically fully replaced by immunofixation $(1,2)$. Immunofixation makes possible a reliable definition of the paraprotein class and antigen type of light chains as well as in „small“ paraproteins $1 \mathrm{~g} / 1$ concentration. The presence of M-protein in the blood serum and/or in urine is relevant for a clinician, but it is not clear whether the detection of very low levels $(<1 \mathrm{~g} / 1)$ of paraprotein is clinically useful (3).

The high sensitivity of immunofixation (about 10 times higher than that of immunoelectrophoresis) leads not only to the identifying the small paraproteins but also the oligoclonal gradients. From time to time we are faced with the dilemma, of whether the gradient should be evaluated as monoclonal or oligoclonal.

In the series of 2413 paraproteins analyzed by immunoelectrophoresis in the years 1967-1995 doubled paraproteinemias were found 42 times, i. e. a frequence of $1.7 \%$ (tab. 1). In the last two years we have found 202 paraproteins by means of immunofixation electrophoresis and within this group multiple paraproteinemias (2-3 paraproteins in the serum of one person) were found 21 times, i. e. a frequence of $10.4 \%$ (tab. 2 and 3). Immunofixation reveals that multiple paraproteinemias are more frequent than had been assumed.

Immunofixation makes the evidence of "ladder" effect easier to detect when the monoclonal light chains are typed
Tab. 1: Immunoglobulin classses in 2413 cases of paraproteinemia (detection of paraproteins by immunoelectrophoresis).

\begin{tabular}{|l|c|c|c|c|c|}
\hline Paraprotein & $\mathrm{n}$ & $\%$ & kappa & lambda & $\begin{array}{c}\text { non } \\
\text { detected }\end{array}$ \\
\hline IgG & 1540 & 63,8 & 854 & 545 & 141 \\
\hline IgA & 396 & 16,4 & 225 & 138 & 33 \\
\hline IgM & 302 & 12,56 & 172 & 85 & 45 \\
\hline IgD & 10 & 0,4 & 1 & 9 & - \\
\hline Light chains & 122 & 5,0 & 70 & 52 & - \\
\hline Gamma HCD & 1 & 0,04 & - & - & - \\
\hline Double paraproteins & 42 & 1,7 & - & - & - \\
\hline
\end{tabular}

Tab. 2: Immunoglobulin classses in 202 cases of paraproteinemia (detection of paraproteins by immunoelectrophoresis).

\begin{tabular}{|l|c|c|c|c|}
\hline Paraprotein & $\mathrm{n}$ & $\%$ & kappa & lambda \\
\hline $\mathrm{IgG}$ & 128 & 63,36 & 78 & 50 \\
\hline $\mathrm{IgA}$ & 22 & 10,9 & 10 & 12 \\
\hline $\mathrm{IgM}$ & 21 & 10,4 & 17 & 4 \\
\hline IgD & 1 & 0,5 & 1 & - \\
\hline Light chains & 9 & 4,45 & 7 & 2 \\
\hline Double paraproteins & 21 & 10,4 & - & - \\
\hline
\end{tabular}

Tab. 3: Multiple paraprotinemias in 202 cases of paraproteinemia (Detection by immunofixation).

\begin{tabular}{|l|c|l|c|}
\hline Paraprotein & $\mathrm{n}$ & Paraprotein & $\mathrm{n}$ \\
\hline IgG-kappa + IgG-lambda & 5 & IgG-lambda + IgM-kappa & 1 \\
\hline IgG-kappa + IgA-lambda & 2 & IgG-kappa + lambda free & 1 \\
\hline IgG-lambda + IgM-lambda & 2 & IgG-kappa + IgG-kappa & 1 \\
\hline IgG-kappa + IgA-kappa & 2 & IgA-lambda + IgM-kappa & 2 \\
\hline IgG-kappa + IgM-kappa & 2 & IgF-kappa + IgA-lambda + & \\
\hline IgG-lambda + IgA-kappa & 1 & IgM-lambda & 1 \\
\hline IgG-lambda + IgAlambda & 1 & & \\
\hline
\end{tabular}


(especially kappa) namely in urine. The interpretation and exact clinical meaning of this phenomenon is not very clear so far.

Modern, sensitive analytical methods used in clinical laboratories to identify and analyze the monoclonal immunoglobulins are an indisputable contribution and improvement in diagnostics, knowledge and monitoring of the therapy of monoclonal gammopathies. It is necessary to solve some of the difficulties of the laboratory evaluation and clinical interpretation of the immunofixation analysis of monoclonal immunoglobulins.

\section{References}

1. Keren DF. Procedures for the evaluation of monoclonal immunoglobulins. Arch Pathol Lab Med 1999(123:126-32.

2. Keren DF, Alexanian R, Goeken JA, Gorevic PD, Kyle RA, Tomar RH Guidelines for clinical and laboratory evaluation of patients with monoclonal gammopathies. Arch Pathol Lab Med 1999(123:106-7.

3. Kyle RA. Sequence of testing for monoclonal gammopathies. Arch Pathol Lab Med 1999(123:114-8.

Submitted July 1999.

Accepted September 1999.

Doc. RNDr. Miloš Tichý, CSc.,

Purkyně Military Medical Academy, Second Department of Internal Medicine, 50005 Hradec Králové, Czech Republic. e-mail: tichy@fnhk.cz 